\title{
Central Venous Line Placement prior to Gastric Bypass Improves Operating Room Efficiency
}

\author{
D. Wayne Overby, ${ }^{1}$ Geoffrey P. Kohn, ${ }^{1}$ Karen J. Colton, ${ }^{1}$ Joseph M. Stavas, ${ }^{2}$ \\ Robert G. Dixon, ${ }^{2}$ Anthony Passannante, ${ }^{3}$ and Timothy M. Farrell ${ }^{1}$ \\ ${ }^{1}$ Department of Surgery, The University of North Carolina at Chapel Hill, Campus Box 7081, Chapel Hill, NC 27599, USA \\ ${ }^{2}$ Department of Radiology, The University of North Carolina at Chapel Hill, Campus Box 7510, Chapel Hill, NC 27599, USA \\ ${ }^{3}$ Department of Anesthesiology, The University of North Carolina at Chapel Hill, Campus Box 7010, Chapel Hill, NC 27599, USA
}

Correspondence should be addressed to Timothy M. Farrell, tfarrell@med.unc.edu

Received 14 February 2012; Accepted 19 April 2012

Academic Editors: A. A. Mangi and J. A. Tovar

Copyright (c) 2012 D. Wayne Overby et al. This is an open access article distributed under the Creative Commons Attribution License, which permits unrestricted use, distribution, and reproduction in any medium, provided the original work is properly cited.

Background. Bariatric surgery has increased across America. Venous access is difficult in these patients. Anesthesiologists often utilize valuable operating room (OR) time acquiring reliable intravenous lines. Our objective was to determine if outpatient central venous line (CVL) placement improves OR efficiency and professional reimbursement for CVL insertion. Methods. In our bariatric practice, selected surgery patients have outpatient CVLs placed during prophylactic vena cava filter placement. In a cohort of 268 gastric bypass patients operated between $1 / 01$ and 11/06, we compared time-to-incision between 106 with pre-established CVLs and 162 without. In addition, we determined professional compensation rates for CVLs placed outpatient versus CVLs inserted in the OR. Results. Patients with preoperative (outpatient) CVLs required $35.6 \pm 12.5$ minutes to skin incision compared with $42.5 \pm 13.9$ minutes for controls $(P<0.0001)$, and $34.9 \%$ had skin incision in $<30$ minutes compared with $16.4 \%$ of controls. Radiologists collected $28.2 \%$ of outpatient billings for CPT code 36556 , compared with anesthesiologists who collected $<1 \%$ when placing CVLs in the OR. Conclusions. Outpatient CVLs prior to gastric bypass improve efficiency in the OR with earlier skin incision. Professional reimbursement is better for outpatient CVLs than intraoperative inpatient CVLs.

\section{Introduction}

Obesity prevalence has increased over the last 20 years [1]. At the turn of the millennium, nearly two-thirds of Americans were overweight or obese, and almost $5 \%$ were morbidly obese [2]. Obesity shortens life expectancy and will likely soon be the leading cause of preventable death in the United States [3].

In the absence of reliable medical and behavioral therapies, and with the advent of minimally invasive technologies, bariatric surgery volumes expanded through 2003 [4-7]. More recent data suggest a leveling in procedure numbers despite the ongoing obesity epidemic [8], perhaps related to the evolution of center-of-excellence requirements $[9$, 10] and linked reimbursement systems [11]. Systems which restrict cases to high-volume centers may ensure better outcomes and reduced costs in certain environments but may also risk stifle small programs by proscribed requirements and limit national bariatric surgery capacity. The current reimbursement environment has set a narrow margin for surgeons and healthcare systems intending to provide stateof-the-art bariatric services [12].

Efficient delivery of operative care is necessary for the financial survival of bariatric programs. Therefore, programs have typically flourished in hospitals which focus on providing well-insured patients efficient customer-centric care [13]. Conversely, programs located in hospitals with prominent charitable and educational missions may struggle to preserve sufficient case volumes to maintain accreditation and profitability due to inherent inefficiencies [14]. 
Global efficiency initiatives for operating room processes have centered on improving case scheduling [15], data tracking [16], staff satisfaction [17], communications at all levels [18-20], and patient outflow [21]. At the provider level, many areas of inefficiency have been improved by simple tracking and delineation of accountability; however, certain patient-related variables, such as late arrival to hospital or difficult venous access, are more resistant to day-of-surgery systems improvement measures [22].

Bariatric surgery patients' body habitus may make achieving venous access challenging. When venous access efforts fail in the holding area, anesthesiologists often utilize valuable OR time acquiring reliable peripheral or central intravenous lines. These services may not be compensated and often impact an anesthesiologist's availability for other scheduled activity.

As a result of the increased prevalence of hypercoagulable states seen in the morbidly obese, we have initiated an aggressive program for venous thromboembolism prophylaxis in our bariatric patients, which includes liberal use of inferior vena cava (IVC) filters for a proportion of our patients who are deemed high risk for pulmonary embolism [23]. Filters are placed in the outpatient setting in the interventional radiology suite $<24$ hours prior to operation, with concomitant central venous line (CVL) insertion in all of these patients. Therefore, since some of our bariatric surgery patients arrive on the day of surgery with reliable venous access and others do not, we have a simple means to assess the effect of reliable venous access on operating room efficiency.

\section{Methods}

The study encompassed 268 consecutive patients who had laparoscopic gastric bypass between January 2001 and November 2006. Of these, 106 were referred to our interventional radiologists and had outpatient preoperative placement of a prophylactic IVC filter $<24$ hours prior to operation. These patients also received outpatient CVLs at the completion of the IVC filter placement.

All patients present through standard same-day-surgery channels on the day of operation. Routine preoperative protocols were applied by administrative, nursing, and physician staff. At University of North Carolina Hospitals, the precare nurses will attempt to achieve peripheral intravenous access'if no access line is present. If venipuncture fails after two attempts, the anesthesiology team assumes responsibility for obtaining primary venous access. Patients are often taken from the holding area to the operating room under the direction of the attending anesthesiologist, and decisions about venous access and monitoring are made at his or her discretion.

We queried operating room databases to compare time between patient OR entry and skin incision ("in-to-skin") for patients with and without outpatient CVLs. In addition, we searched billing databases for CVL collection rates. Since OR benchmarks for "in-to-skin" times are typically stratified by 15-minute increments, we graphically depicted the subject and control data in this fashion. Statistical
TABLE 1

\begin{tabular}{lccc}
\hline & $n$ & Percent female & $\mathrm{BMI} \pm \mathrm{SD}\left(\mathrm{kg} / \mathrm{m}^{2}\right)$ \\
\hline Patients with CVL & 106 & 84 & $51.6 \pm 9.0$ \\
Patients without CVL & 162 & 86 & $50.9 \pm 8.8$ \\
$P$ value & & & NS \\
\hline
\end{tabular}

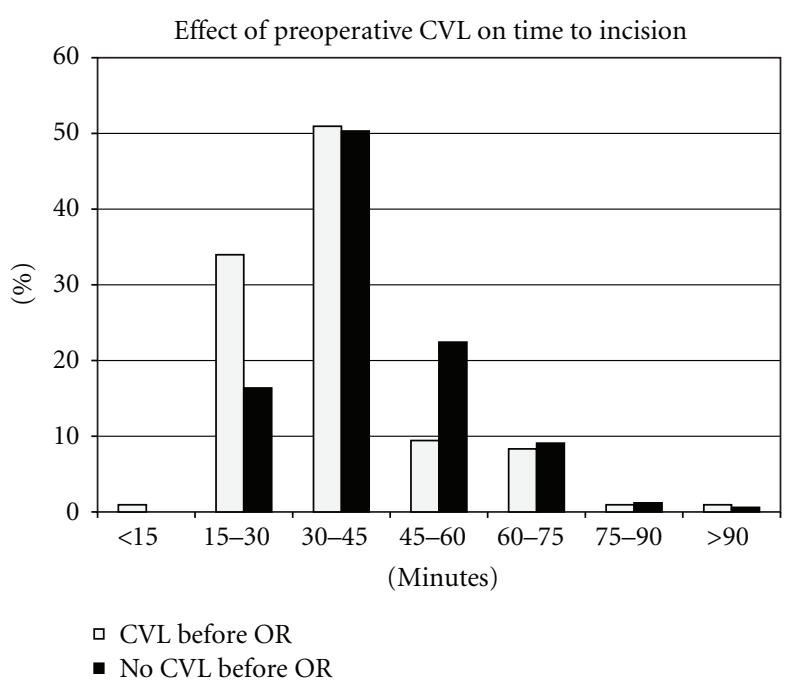

FIGURe 1

comparison utilized the raw continuous data was made by the nonpaired $t$-test, using $P<0.05$ as a measure of statistical significance. Times were described as arithmetic means \pm standard deviations.

\section{Results}

The 106 patients who received preoperative CVLs had demographics and average BMIs similar to the 162 patients who did not receive preoperative CVLs (Table 1).

Patients with preoperative CVLs had mean "in-to-skin" time of $35.6 \pm 12.5$ minutes versus $42.5 \pm 13.9$ minutes for those without preoperative CVLs $(P<0.0001)$. When assessed in quarter-hour increments, the presence of a preoperative CVL was associated with $34.9 \%$ of subjects having skin incision by 30 minutes versus $16.36 \%$ of controls (Figure 1).

Regarding reimbursement, interventional radiologists performing the outpatient CVLs collected $28.2 \%$ of billings for CPT code 36556. In contrast, those anesthesiologists who placed intraoperative CVLs in the operating room collected less than $1 \%$ of dollars billed.

Among those 106 patients who received preoperative CVLs, one catheter-related infection occurred in a patient with a long hospitalization from respiratory failure, and two self-limited catheter site infections were noted after CVL removal. 


\section{Discussion}

While the number of American adults suffering from obesity continues to increase, the annual number of bariatric operations has been stable or decreasing in recent years [8]. This trend may be attributable to the evolution of programs designed to direct patients to certified high-volume centers where the quality of care is believed to be best. With the arrival of a new administration in our nation's capital, America has again focused awareness on prevention, management of chronic disease, and cost containment [24]. Bariatric surgery will likely come to increased attention as the only contemporary option to decrease the burden and overall utilization of healthcare resources related to America's most costly disease. If governmental interventions crystallize candidacy criteria and eliminate bariatric surgery exclusions among healthcare insurers, the resultant resurgence in demand for procedures may tax the capacity of the system to increase patient throughput.

Increasing efficiency of systems is a strategy for increasing delivery of care without a concomitant and proportional increase in cost. In the highly regulated environment of bariatric surgery, efficiency initiatives will no doubt be integral in early efforts to maximally utilize existing systems, at least until the projected rising demand drives further program and infrastructure development.

This study highlights one obvious efficiency intervention related to perioperative intravenous access. We have demonstrated that outpatient CVL placement in planned bariatric patients improves efficiency on the day of surgery. When the effect of a small incremental OR time savings is considered on a broader scale, the potential to achieve significant monetary savings while perhaps even enhancing effective capacity emerges. With case volumes driving all systems for bariatric program certification, such efficiencies may bring many smaller centers to case levels that allow credentials to be obtained, with improved ability to compete for patients and contracts. In addition, improved reimbursement rates for CVL implantation noted in our study will further support the financial success of a program.

Although a higher level of difficulty for intravenous access in morbidly obese patients has not been established in comparative studies, there are a number of references to obesity as a contributing factor in difficult peripheral and central intravenous access [25-31]. Our data suggest that difficult venous access in bariatric surgery patients may contribute to OR delays.

With operating room cost per minute on the order of $\$ 15-25$ [32] and with an average \$66 per minute charged to patients, [33] a savings of 15 minutes per case can have a significant financial impact at the hospital level, especially when a single attending anesthesiologist oversees two, three, or even four rooms at one time. Moreover, each such delay avoided will prevent accumulating costs for subsequent patients' scheduled in the same operating room. Finally, the above estimates of cost do not account for lost efficiencies in terms of underutilized surgeons, operating room and recovery room personnel, and inpatient resources. Since anesthesiologists are reimbursed less than $1 \%$ for CVLs placed in the OR, it is clear that these providers have no financial reason for resisting program initiatives to move CVL placement to the outpatient setting.

In addition to improved OR efficiency, we believe there are likely other intangible benefits to CVL placement prior to gastric bypass. A preexisting CVL reduces the number of tasks that must be performed prior to the induction of general anesthesia, which may decrease frustration levels when dealing with potentially difficult morbidly obese patients. The inpatient nursing and phlebotomy staff also benefit from secure venous access for delivery of medications and blood draws, which decreases their frustration and improves efficiency and customer service during the inpatient stay. Finally, patients are happy to avoid multiple unsuccessful phlebotomy and peripheral venous line (PVL) placement attempts.

There are risks associated with CVLs, including those associated with placement such as arterial puncture, hematoma, and pneumothorax, and those which occur after placement such as catheter-related infections, catheter malfunction, and catheter-related thrombosis. Since the CVL catheters described in this study were placed using ultrasound guidance by experienced vascular interventional radiologists, the insertion complication rate was low $(0.62 \%)$ [34], and since the majority of patients were immune competent, not critically ill, and had brief CVL dwell times, low rates of catheter-related infection were seen. In fact, similar risks have been associated with PVLs, including arterial puncture, hematoma, infiltration, catheter malfunction, and catheter-related infections. While the rate of minor complications such as infiltration or phlebitis is high with PVLs (up to 21.7\%) [35], the rate of serious complications such as bacteremia is probably lower when compared to CVLs.

Anecdotal reports of increased patient and anesthesiologist satisfaction with CVLs are being assessed in subsequent studies. Additional implementation of a plan for increased CVL utilization to enhance OR efficiency will need to be balanced against the impact on interventional radiology unit capacity and professional satisfaction of the affected radiologists, as well as the impact on trainees in the operating rooms.

One drawback of the current study is its retrospective nature. Useful data regarding OR efficiency, related costs, potential savings, and complications could be obtained from a prospective randomized control trial comparing CVL placement prior to gastric bypass to CVL placement at the time of gastric bypass to peripheral intravenous access at the time of gastric bypass.

\section{Conclusions}

Outpatient placement of CVLs prior to gastric bypass improves the efficiency of the operating room with earlier skin incision. For patients having IVC filter placement prior to bariatric surgery, concomitant CVL placement is advised.

For those patients not referred for IVC placement, we suggest surgeons and anesthesiologists evaluate for difficult intravenous access during the outpatient preoperative visit and consider referral for ultrasound-guided access during 
the 24 hours prior to scheduled surgery. Such efforts will improve day-of-surgery efficiency and professional reimbursement for necessary procedures.

\section{References}

[1] C. L. Ogden, C. D. Fryar, M. D. Carroll, and K. M. Flegal, "Mean body weight, height, and body mass index, United States 1960-2002," Advance Data, no. 347, pp. 1-17, 2004.

[2] K. M. Flegal, M. D. Carroll, C. L. Ogden, and C. L. Johnson, "Prevalence and trends in obesity among US adults, 19992000," Journal of the American Medical Association, vol. 288, no. 14, pp. 1723-1727, 2002.

[3] D. B. Allison, K. R. Fontaine, J. E. Manson, J. Stevens, and T. B. VanItallie, "Annual deaths attributable to obesity in the United States," Journal of the American Medical Association, vol. 282, no. 16, pp. 1530-1538, 1999.

[4] B. M. Wolfe and J. M. Morton, "Weighing in on bariatric surgery: procedure use, readmission rates, and mortality," Journal of the American Medical Association, vol. 294, no. 15, pp. 1960-1963, 2005.

[5] D. M. Herron and R. Bloomberg, "Complications of bariatric surgery," Minerva Chirurgica, vol. 61, no. 2, pp. 125-139, 2006.

[6] J. L. Sebastian, "Bariatric surgery and work-up of the massive weight loss patient," Clinics in Plastic Surgery, vol. 35, no. 1, pp. 11-26, 2008.

[7] D. Leslie, T. A. Kellogg, and S. Ikramuddin, "Bariatric surgery primer for the Internist: keys to the surgical consultation," Medical Clinics of North America, vol. 91, no. 3, pp. 353-381, 2007.

[8] G. P. Kohn, J. A. Galanko, D. W. Overby, and T. M. Farrell, "Recent trends in bariatric surgery case volume in the United States," Surgery, vol. 146, no. 2, pp. 375-380, 2009.

[9] B. Schirmer and D. B. Jones, "The American College of Surgeons Bariatric Surgery Center Network: establishing standards," Bulletin of the American College of Surgeons, vol. 92, no. 8, pp. 21-27, 2007.

[10] http://www.surgicalreview.org/.

[11] http://www.cms.hhs.gov/apps/media/press/release.asp? Counter $=1786$.

[12] A. K. Madan, J. E. Powelson, and D. S. Tichansky, "Cost analysis of laparoscopic gastric bypass practice using current Medicare reimbursement and practice costs," Surgery for Obesity and Related Diseases, vol. 4, no. 2, pp. 131-136, 2008.

[13] A. K. Madan, D. S. Tichansky, C. A. Ternovits et al., "Establishing a laparoscopic bariatric program in a safety net hospital," Surgical Endoscopy and Other Interventional Techniques, vol. 21, no. 5, pp. 801-804, 2007.

[14] E. H. Livingston, A. C. Elliott, L. S. Hynan, and E. Engel, "When policy meets statistics: the very real effect that questionable statistical analysis has on limiting health care access for bariatric surgery," Archives of Surgery, vol. 142, no. 10, pp. 979-987, 2007.

[15] I. H. Wright, C. Kooperberg, B. A. Bonar, and G. Bashein, "Statistical modeling to predict elective surgery time: comparison with a computer scheduling system and surgeon-provided estimates," Anesthesiology, vol. 85, no. 6, pp. 1235-1245, 1996.

[16] P. G. Nagy, R. Konewko, M. Warnock et al., "Novel, web-based, information-exploration approach for improving operating room logistics and system processes," Surgical Innovation, vol. 15, no. 1, pp. 7-16, 2008.

[17] M. J. Heslin, B. E. Doster, S. L. Daily et al., "Durable improvements in efficiency, safety, and satisfaction in the operating room," Journal of the American College of Surgeons, vol. 206, no. 5, pp. 1083-1089, 2008.

[18] R. G. Soto, L. F. Chu, J. M. Goldman, I. J. Rampil, and K. J. Ruskin, "Communication in critical care environments: mobile telephones improve patient care," Anesthesia and Analgesia, vol. 102, no. 2, pp. 535-541, 2006.

[19] S. Nundy, A. Mukherjee, J. B. Sexton et al., "Impact of preoperative briefings on operating room delays: a preliminary report," Archives of Surgery, vol. 143, no. 11, pp. 1068-1072, 2008.

[20] J. F. Cosgrove, M. Gaughan, C. P. Snowden, and T. Lees, "Decreasing delays in urgent and expedited surgery in a university teaching hospital through audit and communication between peri-operative and surgical directorates," Anaesthesia, vol. 63, no. 6, pp. 599-603, 2008.

[21] J. Jenkins, "Eliminating common PACU delays," Journal of Healthcare Information Management, vol. 21, no. 2, pp. 53-58, 2007.

[22] F. J. Overdyk, S. C. Harvey, R. L. Fishman, and F. Shippey, "Successful strategies for improving operating room efficiency at academic institutions," Anesthesia and Analgesia, vol. 86, no. 4, pp. 896-906, 1998.

[23] D. W. Overby, G. P. Kohn, M. A. Cahan et al., "Risk-group targeted inferior vena cava filter placement in gastric bypass patients," Obesity Surgery, vol. 19, no. 4, pp. 451-455, 2009.

[24] B. Obama, June 2009, http://www.whitehouse.gov/the_press_ office/Letter-from-President-Obama-to-Chairmen-EdwardM-Kennedy-and-Max-Baucus/.

[25] R. Blackshaw, W. Peat, and P. Youngs, "Use of ultrasound to obtain peripheral venous access," International Journal of Obstetric Anesthesia, vol. 15, no. 2, pp. 174-175, 2006.

[26] C. D. Botkin and M. M. Osman, "Prevalence, challenges, and solutions for 18F-FDG PET studies of obese patients: a technologist's perspective," Journal of Nuclear Medicine Technology, vol. 35, no. 2, pp. 80-83, 2007.

[27] L. Brannam, M. Blaivas, M. Lyon, and M. Flake, "Emergency nurses' utilization of ultrasound guidance for placement of peripheral intravenous lines in difficult-access patients," Academic Emergency Medicine, vol. 11, no. 12, pp. 1361-1363, 2004.

[28] T. G. Costantino, A. K. Parikh, W. A. Satz, and J. P. Fojtik, "Ultrasonography-guided peripheral intravenous access versus traditional approaches in patients with difficult intravenous access," Annals of Emergency Medicine, vol. 46, no. 5, pp. 456-461, 2005.

[29] L. E. Keyes, B. W. Frazee, E. R. Snoey, B. C. Simon, and D. Christy, "Ultrasound-guided brachial and basilic vein cannulation in emergency department patients with difficult intravenous access," Annals of Emergency Medicine, vol. 34, no. 6, pp. 711-714, 1999.

[30] U. Mey, A. Glasmacher, C. Hahn et al., "Evaluation of an ultrasound-guided technique for central venous access via the internal jugular vein in 493 patients," Supportive Care in Cancer, vol. 11, no. 3, pp. 148-155, 2003.

[31] E. Ottestad, C. Schmiessing, J. G. Brock-Utne, V. Kulkarni, D. Parris, and J. B. Brodsky, "Central venous access in obese patients: a potential complication," Anesthesia and Analgesia, vol. 102, no. 4, pp. 1293-1294, 2006.

[32] J. E. Stahl, W. S. Sandberg, B. Daily et al., "Reorganizing patient care and workflow in the operating room: a costeffectiveness study," Surgery, vol. 139, no. 6, pp. 717-728, 2006.

[33] R. D. Shippert, "A study of time-dependent operating room fees and how to save $\$ 100000$ by using time-saving devices," The American Journal of Cosmetic Surgery, vol. 22, p. 10, 2005. 
[34] D. W. Overby, G. P. Kohn, M. A. Cahan et al., "Risk-group targeted inferior vena cava filter placement in gastric bypass patients," Obesity Surgery, vol. 19, no. 4, pp. 451-455, 2009.

[35] N. E. Soifer, S. Borzak, B. R. Edlin, and R. A. Weinstein, "Prevention of peripheral venous catheter complications with an intravenous therapy team: a randomized controlled trial," Archives of Internal Medicine, vol. 158, no. 5, pp. 473-477, 1998. 


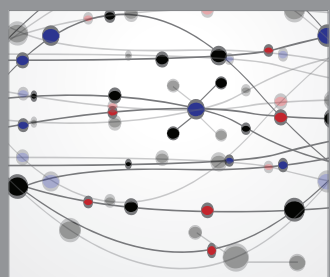

The Scientific World Journal
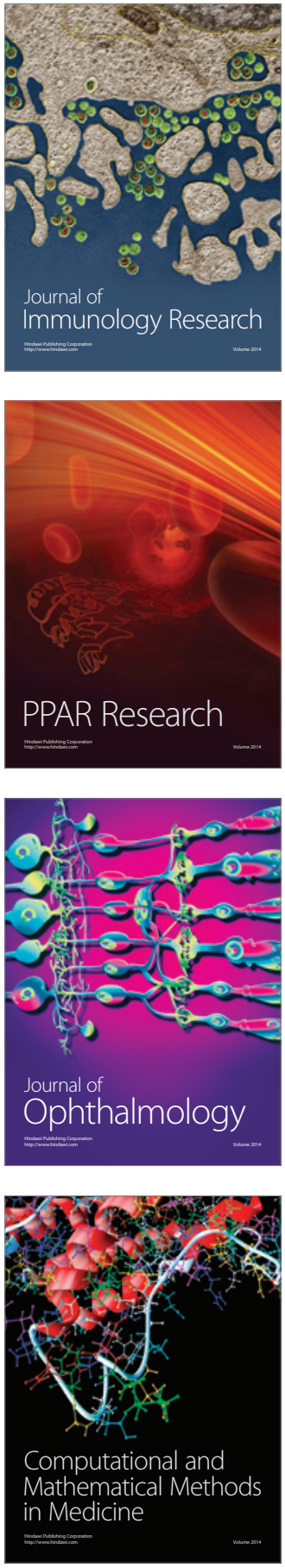

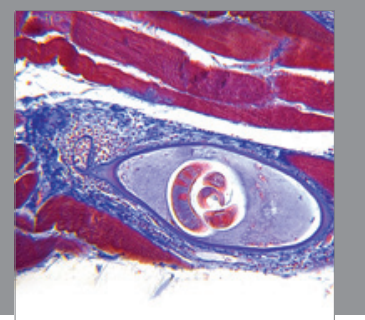

Gastroenterology

Research and Practice
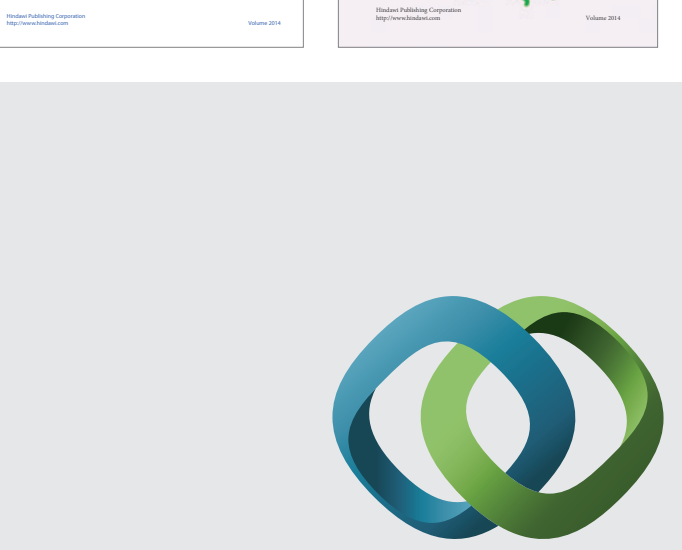

\section{Hindawi}

Submit your manuscripts at

http://www.hindawi.com
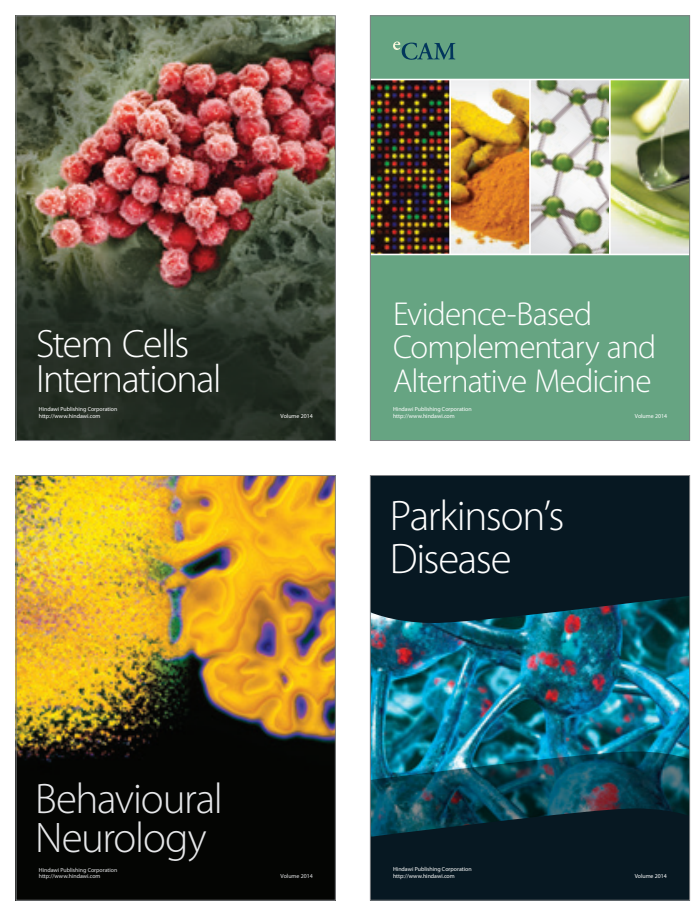

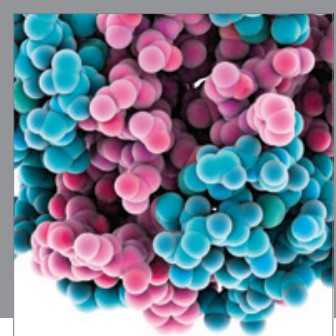

Journal of
Diabetes Research

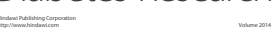

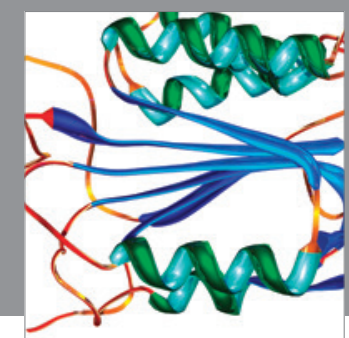

Disease Markers
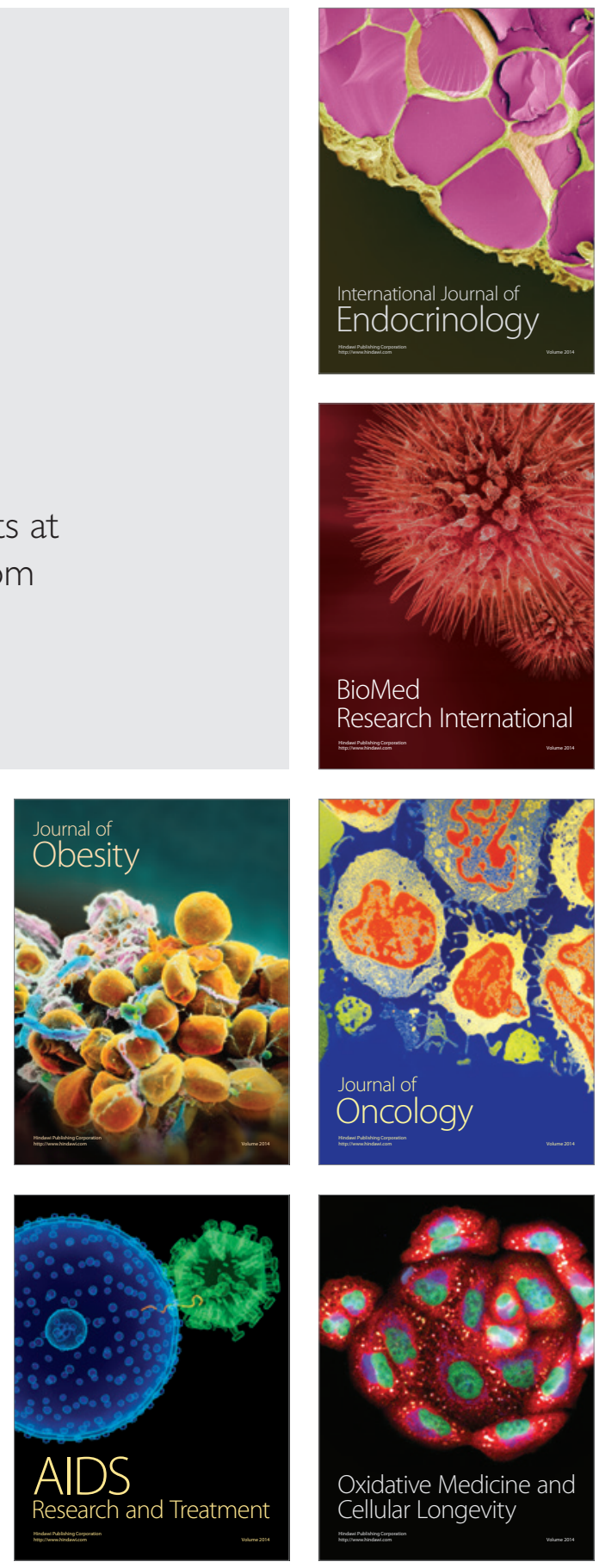